\title{
Transient bilateral abducens neuropathy with post-tetanic facilitation and acute hypokalemia associated with oxaliplatin: a case report
}

\author{
Min-Han Tan ${ }^{1,2^{*}}$, Wen Yee Chay ${ }^{1}$, Jia Hui $\mathrm{Ng}^{1}$, Bin Tean Teh², Lita Chew ${ }^{3}$
}

\begin{abstract}
Introduction: Oxaliplatin is a cytotoxic platinum compound that is in widespread use in the treatment of gastrointestinal cancers. It has been occasionally associated with acute motor neuropathy, but the precise mechanism is uncertain. To the best of our knowledge, we report the first case of a patient demonstrating posttetanic facilitation in the setting of transient bilateral abducens neuropathy and hypokalemia, after being infused with oxaliplatin.

Case presentation: A 47-year-old Indian woman with metastatic gastric cancer was receiving an oxaliplatin infusion at the initiation of her third cycle of palliative chemotherapy. She developed acute bilateral abducens neuropathy with post-tetanic facilitation alongside acute laryngopharyngodysesthesia and hypokalemia. Following supportive management, including potassium infusion and warming, her neurological signs and symptoms were spontaneously resolved. This syndrome did not recur in subsequent cycles following prolongation of infusion duration and the addition of supportive calcium and magnesium infusions.
\end{abstract}

Conclusion: The novel clinical observation of post-tetanic facilitation highlights a possible involvement of voltagegated channels at the presynaptic terminals in the mechanism of acute oxaliplatin neurotoxicity.

\section{Introduction}

Oxaliplatin is a recently developed cytotoxic platinum compound that is in widespread use, particularly for the treatment of gastrointestinal cancers [1]. The association between oxaliplatin and acute and chronic sensory neuropathy is well recognized, but acute motor neuropathy is also reported, albeit much less frequently [2]. The exact mechanisms of both acute and chronic neurotoxicity remain uncertain, despite extensive clinical experience with the compound. It is conventionally regarded that the voltage-gated sodium channels are involved in mechanisms of acute neurotoxicity [3], possibly through a pathway involving calcium ions [4]. The use of calcium and magnesium infusions is currently under investigation [5].

\section{Case presentation}

We report a novel observation in a 47-year-old Indian woman with no comorbidities who developed acute

\footnotetext{
* Correspondence: tan.min.han@nccs.com.sg

'Department of Medical Oncology, National Cancer Centre, 11 Hospital Drive, Singapore 169610, Republic of Singapore
}

\section{.}

bilateral abducens neuropathy with post-tetanic facilitation alongside acute hypokalemia after infusion of oxaliplatin. She was first diagnosed with peritoneal carcinomatosis arising from metastatic gastric adenocarcinoma, and was commenced on a palliative chemotherapy regimen of continuous infusion 5-fluorouracil (5FU) $200 \mathrm{mg} / \mathrm{m}^{2} /$ day and weekly oxaliplatin $(50 \mathrm{mg}$ absolute dose). After the first cycle totaling $150 \mathrm{mg}$ oxaliplatin, during which significant clinical and radiological improvement in her intestinal function was documented permitting administration of oral medication, her regimen was modified to a three-weekly regimen of oxaliplatin $\left(130 \mathrm{mg} / \mathrm{m}^{2}\right.$ on day 1 over 2 hours) and capecitabine $\left(2000 \mathrm{mg} / \mathrm{m}^{2} /\right.$ day on day 1 evening - day 15 morning) (XELOX). One hour into the infusion of oxaliplatin of the 2nd cycle of XELOX, she developed acute neuropathy comprising acute bilateral abducens neuropathy, dysarthria, laryngopharyngodysesthesia, perioral and peripheral numbness. In addition to oxaliplatin, she had received $8 \mathrm{mg}$ of intravenous dexamethasone and $8 \mathrm{mg}$ of intravenous ondansetron as

C 2010 Tan et al; licensee BioMed Central Ltd. This is an Open Access article distributed under the terms of the Creative Commons BioMed Central Attribution License (http://creativecommons.org/licenses/by/2.0), which permits unrestricted use, distribution, and reproduction in any medium, provided the original work is properly cited. 
premedication. The patient had not taken any oral medications other than capecitabine up to one week previously. The patient demonstrated physical signs consistent with bilateral abducens neuropathy, with bilateral gaze-evoked diplopia. Surprisingly, post-tetanic facilitation was observed, with unilateral resolution of diplopia and abducens neuropathy following sustained lateral gaze over one minute. Contralateral abducens neuropathy remained unchanged following apparent resolution of the unilateral abducens neuropathy. The apparently resolved unilateral abducens neuropathy recurred following a two-minute rest. Decreased tendon reflexes diffusely were noted, but no focal or generalized weakness was found. New electrocardiographic changes in terms of diffuse $\mathrm{T}$-wave inversions were noted, as well as mild elevation of her serum creatine kinase-MB (CK-MB) levels by mass assay. Also noted was a serial decrease over 16 hours $(7.0 \mu \mathrm{g} / \mathrm{L}$ immediately, $5.2 \mu \mathrm{g} / \mathrm{L}$ 8 hours later and $3.9 \mu \mathrm{g} / \mathrm{L} 16$ hours later), but the diffuse $\mathrm{T}$-wave inversions persisted. Corresponding serial serum creatine kinase (CK) levels were $72 \mu \mathrm{g} / \mathrm{L}, 63 \mu \mathrm{g} / \mathrm{L}$ and $52 \mu \mathrm{g} / \mathrm{L}$, and troponin-T levels were not elevated at any of these three-time points.

Baseline blood counts and electrolytes drawn two days prior were unremarkable; in particular, her potassium level was $3.5 \mathrm{mmol} \mathrm{L}^{-1}\left(3.3-4.9 \mathrm{mmol} \mathrm{L}^{-1}\right)$. She was asymptomatic up till this event. In particular, there was no intervening symptom of vomiting or diarrhoea, and the patient did not report the use of any oral medications. The patient did not have prior hypokalemia up to this point. Electrolytes drawn immediately upon onset of neurological symptoms revealed acute hypokalemia, with a serum potassium level of $2.5 \mathrm{mmol} \mathrm{L}^{-1}$ with a normal serum bicarbonate level of $23.1 \mathrm{mmol} \mathrm{L}^{-1}(19.0-31.0$ $\left.\mathrm{mmol} \mathrm{L} \mathrm{L}^{-1}\right)$, normal corrected serum total calcium level of 2.28 (2.10-2.60 $\left.\mathrm{mmol} \mathrm{L}^{-1}\right)$ and magnesium level of $0.78 \mathrm{mmol} / \mathrm{L}\left(0.70-0.95 \mathrm{mmol} \mathrm{L}^{-1}\right)$.

The patient was placed in a warm environment and an aggressive potassium replacement was undertaken. She did not receive calcium or magnesium infusions. The bilateral abducens neuropathy, dysarthria and laryngopharyngodysesthesia resolved over two hours. The hypokalemia, perioral and peripheral numbness resolved over twelve hours. Due to the short duration of the episode, no electromyogram (EMG) or nerve conduction testing was performed. The diffuse T-wave inversions on ECG did not resolve and remained six months following this event.

The patient was re-challenged with the same dose of oxaliplatin for her fourth cycle, with infusion time extended from 2 to 6 hours, together with calcium and magnesium infusions, and did not experience subsequent neurotoxicity. She continued to receive secondline irinotecan and cisplatin, third-line docetaxel, fourth- line epirubicin, cisplatin and 5-fluorouracil and fifth line 5-fluorouracil and cetuximab. All treatments were tolerated reasonably without further adverse events. The patient eventually passed away from progressive disease approximately fifteen months after initial diagnosis while on best supportive care.

\section{Discussion}

While the mechanism of oxaliplatin neurotoxicity remains uncertain, EMG studies have demonstrated peripheral motor nerve hyperexcitability coinciding with symptoms of acute neuropathy, including cold-induced paresthesias and larygopharyngodysesthesias [6]. It is conventionally regarded that voltage-gated sodium channels are involved. Preclinical studies showed that oxaliplatin causes prolonged opening of voltage-gated sodium channels in sensory nerves with a resulting hyperexcitable state [7]. It has been suggested in preclinical studies that oxaliplatin may mediate this channelopathy through the rapid chelation of unbound calcium through its oxalate metabolite [4].

A previous ex vivo study investigating voltage-gated potassium channels using blockers such as apamin has been performed, with no apparent correlation between phenotypes induced by apamin and acute oxaliplatin neurotoxicity [8]. The authors, however, argue that a population of apamin-resistant, calcium-dependent potassium channels has been identified, and that calcium chelation by oxalate provides a viable mechanism for this to occur. Indeed, recently reported ex vivo work suggests that oxaliplatin may interfere with voltagegated potassium channels $[9,10]$. Unexpectedly, one of these two reports did not detect an effect of oxaliplatin on voltage-gated sodium channels [10].

A single case report previously has reported an association between oxaliplatin and bilateral abducens nerve palsy in a patient heavily exposed to previous cisplatin [11], but did not report on the phenomenon of posttetanic facilitation or associated hypokalemia. Post-tetanic facilitation has not been previously recognized, and may imply the involvement of voltage-gated channels in the presynaptic terminal, where both potassium and calcium channels are present. Calcium-activated presynaptic potassium channels in Xenopus are known to regulate transmitter release magnitude during single action potentials by altering the rate of action potential repolarization, and thus the magnitude of peak calcium current [12].

The acute hypokalemia we observe has been reported in a separate case report where a coma associated with hypokalemia and hypocalcemia was reported after infusion of oxaliplatin [13]. The mechanism is uncertain. Given that our patient presented with hypokalemia but not hypocalcemia, the data does not support systemic 
calcium chelation as the primary mechanism of acute oxaliplatin-induced neurotoxicity, but it is conceivable that a specific locations along the peripheral nerve may be more vulnerable to accumulation of oxaliplatin or its metabolites [1]. For example, platinum has been shown to accumulate in dorsal root ganglia in rodents administered oxaliplatin.

We considered the possibility of the acute hypokalemia causing the post-tetanic facilitation that we observed. Acute hypokalemia is associated with axonal and muscle membrane hyperpolarization $[14,15]$. It must be acknowledged that although our review of the literature did not report a previous association between hypokalemia and post-tetanic facilitation, the mechanisms of hypokalemia in inducing weakness are complex and as-yet poorly understood. Hence, a possible contribution of hypokalemia to post-tetanic facilitation cannot be definitively excluded. However, recent electrophysiologic investigations of patients with acute hypokalemia highlight its contribution to axonal hyperpolarization, with a resulting activity-dependent conduction block worsening, rather than improving weakness [14]. Hence, this data suggests that hypokalemia is not the primary mechanism for post-tetanic facilitation.

\section{Conclusion}

In summary, while the mechanism of acute oxaliplatininduced neuropathy remains uncertain, our novel clinical observation of post-tetanic facilitation alongside acute hypokalemia highlights voltage-gated channels at the presynaptic nerve terminal for investigation in the mechanism of acute oxaliplatin neurotoxicity.

\section{Consent}

Written informed consent was obtained from the patient's family for publication of this case report. A copy of the written consent is available for review by the Editor-in-Chief of this journal.

\section{Acknowledgements}

We would like to thank Dr. Umapathi Thirugnanam and Dr. Kevin Tan of the Department of Neurology, National Neuroscience Institute for their discussion and input. We would also like to thank the family of the patient for consenting to publication. MHT is supported by the Singapore Millenium Foundation and the National Kidney Foundation.

\footnotetext{
Author details

'Department of Medical Oncology, National Cancer Centre, 11 Hospital Drive, Singapore 169610, Republic of Singapore. ${ }^{2}$ NCCS-VARI Laboratory of Translational Cancer Research, National Cancer Centre, 11 Hospital Drive, Singapore 169610, Republic of Singapore. ${ }^{3}$ Oncology Pharmacy, National Cancer Centre, 11 Hospital Drive, Singapore 169610, Republic of Singapore.
}

\section{Authors' contributions}

MHT wrote the manuscript. JHN and LC obtained data and reviewed the literature. CWY and BTT helped write the manuscript. All authors read and approved the final manuscript.

\section{Competing interests}

The authors declare that they have no competing interests.

Received: 4 November 2009

Accepted: 2 February 2010 Published: 2 February 2010

\section{References}

1. Cersosimo RJ: Oxaliplatin-associated neuropathy: a review. Ann Pharmacother 2005, 39:128-135.

2. Argyriou AA, Polychronopoulos P, Iconomou G, Chroni E, Kalofonos HP: A review on oxaliplatin-induced peripheral nerve damage. Cancer Treat Rev 2008, 34:368-377.

3. Park SB, Goldstein D, Lin CS, Krishnan AV, Friedlander ML, Kiernan MC: Acute Abnormalities of Sensory Nerve Function Associated With Oxaliplatin-Induced Neurotoxicity. J Clin Oncol 2009, 27:1243-1249.

4. Grolleau F, Gamelin L, Boisdron-Celle M, Lapied B, Pelhate M, Gamelin E: A possible explanation for a neurotoxic effect of the anticancer agent oxaliplatin on neuronal voltage-gated sodium channels. J Neurophysiol 2001, 85:2293-2297.

5. Gamelin L, Boisdron-Celle M, Morel A, Poirier AL, Berger V, Gamelin E, Tournigand $C$, de Gramont A: Oxaliplatin-related neurotoxicity: interest of calcium-magnesium infusion and no impact on its efficacy. $J$ Clin Oncol 2008, 26:1188-1189.

6. Wilson RH, Lehky T, Thomas RR, Quinn MG, Floeter MK, Grem JL: Acute oxaliplatin-induced peripheral nerve hyperexcitability. J Clin Oncol 2002, 20:1767-1774.

7. Adelsberger H, Quasthoff S, Grosskreutz J, Lepier A, Eckel F, Lersch C: The chemotherapeutic oxaliplatin alters voltage-gated $\mathrm{Na}(+)$ channel kinetics on rat sensory neurons. Eur J Pharmacol 2000, 406:25-32.

8. Webster RG, Brain KL, Wilson RH, Grem JL, Vincent A: Oxaliplatin induces hyperexcitability at motor and autonomic neuromuscular junctions through effects on voltage-gated sodium channels. Br J Pharmacol 2005, 146:1027-1039.

9. Benoit E, Brienza S, Dubois JM: Oxaliplatin, an anticancer agent that affects both $\mathrm{Na}+$ and $\mathrm{K}+$ channels in frog peripheral myelinated axons. Gen Physiol Biophys 2006, 25:263-276.

10. Kagiava A, Tsingotjidou A, Emmanouilides C, Theophilidis G: The effects of oxaliplatin, an anticancer drug, on potassium channels of the peripheral myelinated nerve fibres of the adult rat. Neurotoxicology 2008, 29:1100-1106

11. Winquist $E$, Vincent $M$, Stadler $W$ : Acute bilateral abducens paralysis due to oxaliplatin. J Natl Cancer Inst 2003, 95:488-489.

12. Pattillo JM, Yazejian B, DiGregorio DA, Vergara JL, Grinnell AD, Meriney SD: Contribution of presynaptic calcium-activated potassium currents to transmitter release regulation in cultured Xenopus nerve-muscle synapses. Neuroscience 2001, 102:229-240.

13. Basso M, Cassano A, Modoni A, Spada D, Trigila N, Quirino M, Schinzari G, Barone C: A reversible coma after oxaliplatin administration suggests a pathogenetic role of electrolyte imbalance. Eur J Clin Pharmacol 2008, 64:739-741.

14. Krishnan AV, Colebatch JG, Kiernan MC: Hypokalemic weakness in hyperaldosteronism: activity-dependent conduction block. Neurology 2005, 65:1309-1312.

15. Kuwabara S, Kanai K, Sung JY, Ogawara K, Hattori T, Burke D, Bostock H: Axonal hyperpolarization associated with acute hypokalemia: multiple excitability measurements as indicators of the membrane potential of human axons. Muscle Nerve 2002, 26:283-287.

doi:10.1186/1752-1947-4-36

Cite this article as: Tan et al:: Transient bilateral abducens neuropathy with post-tetanic facilitation and acute hypokalemia associated with oxaliplatin: a case report. Journal of Medical Case Reports 2010 4:36. 